\title{
Macrophage-conditioned medium upregulates the expression of cannabinoid receptors in human preadipocytes
}

\author{
P. Gonzalez-Muniesa, C. Bing and P. Trayhurn \\ Obesity Biology Research Unit, School of Clinical Sciences, University of Liverpool, Liverpool L69 3GA, UK
}

Obesity is characterised by chronic low-grade inflammation. In adipose tissue adipocytes are directly involved in the inflammatory response and macrophages, which infiltrate the tissue in obesity, also play a major role ${ }^{(1,2)}$. The endocannabinoid system (ECS) has been proposed as a regulator of inflammatory (anti-inflammatory) and angiogenic processes ${ }^{(3,4)}$. There are two major cannabinoid receptors, CB1 and CB2, while both TRPV1 and GPR55 have been considered as a putative third receptor ${ }^{(5,6)}$. The aim of the present study was to determine whether macrophages modulate the expression of the different cannabinoid receptors in human adipocytes and preadipocytes, which was examined by treating preadipocytes and adipocytes with macrophage-conditioned medium.

Human subcutaneous preadipocytes (Zen-Bio Inc., Research Triangle Park, NC, USA) were used as the primary source of human preadipocytes or adipocytes. Conditioned medium was obtained from active U937 cells (a macrophage-like cell line). RT-PCR was used to define the expression of the ECS receptors in different cell types within adipose tissue. Gene expression was quantified by real-time PCR.

Cells were fed for $20 \mathrm{~h}$ with $1 \mathrm{ml}$ medium containing $150 \mu \mathrm{l}$ conditioned- or unconditioned-macrophage medium (control group). The adipocytes incubated with conditioned medium showed an up-regulation in the expression of key inflammatory markers IL-1 $\beta$ (55-fold), IL-6 (30-fold), monocyte chemoattractant protein-1 (MCP-1; 19-fold) and TNFo (3.5-fold) and angiogenic factors vascular endothelial growth factor (VEGF; 5-fold) and angiopoietin-like protein 4 (ANGPTL4; 2.4-fold). However, there were no significant effects on CB1 and TRPV1 mRNA levels in mature adipocytes. Preadipocytes, after the same treatment, showed an increase in the expression of IL-1 $\beta$ (145-fold), IL-6 (20-fold), MCP-1 (31-fold) and ANGPTL4 (2.4-fold), but not in TNF $\alpha$ and VEGF expression. In contrast to adipocytes, in preadipocytes the conditioned medium led to an increase in CB1 and TRPV1 expression (3-fold and 4.5-fold respectively).

These results show that while macrophage-conditioned medium leads to an up-regulation of the expression of genes encoding inflammatory and angiogenic markers in both adipocytes and preadipocytes, a stimulatory effect on cannabinoid receptor gene expression is evident only in preadipocytes. This finding might indicate that while the recruitment of macrophages into adipose tissue generally augments a pro-inflammatory state, increased cannabinoid receptor expression in preadipocytes may be part of a counter-regulatory antiinflammatory response. The cross talk between different cell types within adipose tissue is likely to be both extensive and complex.

We are grateful to Adrian O'Hara for providing macrophage-conditioned medium. This work was funded by a grant from the Biotechnology and Biological Sciences Research Council.

1. Weisberg SP, McCann D, Desai M et al. (2003) J Clin Invest 112, 1796-1808.

2. Xu H, Barnes GT, Yang Q et al. (2003). J Clin Invest 112, 1821-1830.

3. Szallasi A, Cortright DN, Blum CA et al. (2007) Nat Rev Drug Discov 6, 357-372.

4. Di Marzo V (2008) Nat Rev Drug Discov 7, 438-455.

5. Brown AJ (2007) Br J Pharmacol 152, 567-575.

6. Pertwee RG (2007) Br J Pharmacol 152, 984-986. 